\title{
COMPETIÇÃO LARVAL EM Chrysomya megacephala (F.) (DIPTERA: CALLIPHORIDAE): EFEITOS DE DIFERENTES NÍVEIS DE AGREGAÇÃO LARVAL SOBRE ESTIMATIVAS DE PESO, FECUNDIDADE E INVESTIMENTO REPRODUTIVO
}

\author{
VON ZUBEN, C. J., ${ }^{1,4}$ STANGENHAUS, G. ${ }^{2}$ e GODOY, W. A. C. ${ }^{3}$ \\ ${ }^{1}$ Departamento de Zoologia, Instituto de Biociências, Universidade Estadual Paulista, C.P. 199, \\ CEP 13506-900, Rio Claro, SP, Brasil \\ ${ }^{2}$ Departamento de Estatística, Instituto de Matemática, Estatística e Ciências da Computação, Universidade \\ Estadual de Campinas, C.P. 6065, CEP 13083-970, Campinas, SP, Brasil \\ ${ }^{3}$ Departamento de Parasitologia, IB, Universidade Estadual Paulista, Campus de Botucatu, CEP 18618-000, \\ Botucatu, SP, Brasil \\ ${ }^{4}$ Bolsista do $\mathrm{CNPq}$ \\ Correspondência para: Claudio J. Von Zuben, Departamento de Zoologia, Instituto de Biociências, \\ Universidade Estadual Paulista, C.P. 199, CEP 13506-900, Rio Claro, SP, Brasil \\ Recebido em 04/03/98 - Aceito em 10/02/2000 - Distribuído em 31/05/2000
}

\begin{abstract}
Larval competition in Chrysomya megacephala (F.) (Diptera: Calliphoridae): effects of different levels of larval aggregation on estimates of weight, fecundity and reproductive investment
\end{abstract}

In insects that utilize patchy and ephemeral resources for feeding and egg laying, the outcome of larval competition for food resources depends on the amount of resources and the spatial distribution of immatures among patches of food. In the present study, the results of larval competition for food in Chrysomya megacephala, in traits such as female weight, fecundity and reproductive investment, were different in situations where the level of larval aggregation (proportion of competitors per amount of food) was the same, but with densities of competitors and amounts of food proportionally different. These results are indicative that the larval competition may depend both on the larval density and the amount of food, in different situations with the same proportion of larvae per gram of food.

Key words: Chrysomya megacephala, fecundity, female weight, larval aggregation, reproductive investment.

\section{RESUMO}

Em insetos que se utilizam de substratos discretos e efêmeros para a alimentação e postura de ovos, o resultado da competição larval por alimento depende da quantidade de recursos e da distribuição espacial dos imaturos nos substratos alimentares. No presente estudo, os resultados da competição larval por alimento em Chrysomya megacephala, em caracteres como peso de fêmeas, fecundidade e investimento reprodutivo, foram diferentes em situações em que o nível de agregação larval (proporção de competidores por quantidade de alimento) é o mesmo, mas com densidades de competidores e quantidades de alimento proporcionalmente diferentes. Esses resultados indicam que a competição larval pode depender tanto da densidade larval como da quantidade de alimento, em situações diferentes com a mesma proporção de larvas por grama de alimento.

Palavras-chave: Chrysomya megacephala, fecundidade, peso de fêmeas, agregação larval, investimento reprodutivo. 


\section{INTRODUÇÃO}

Chrysomya megacephala (F.) é uma espécie de mosca-varejeira recentemente introduzida no Brasil (Guimarães et al., 1978; Laurence, 1981), de considerável importância médico-sanitária, podendo ser vetora de microrganismos patogênicos ao homem (Furlanetto et al., 1984), causar mí́ases facultativas nos animais e no homem (Guimarães et al., 1983; Hall \& Wall, 1995), bem como ser utilizada como indicadora forense em estudos de medicina legal (Greenberg, 1991; Wells \& Kurahashi, 1994).

A exemplo de muitas espécies de moscasvarejeiras, C. megacephala utiliza substratos discretos e efêmeros, como, por exemplo, carcaças em decomposição e fezes, para alimentação e postura dos ovos (Kamal, 1958; Putman, 1977; Hanski, 1987b). O contexto espacial em que ocorre competição por recursos alimentares em moscas-varejeiras caracteriza-se pela divisão de recursos no ambiente, sendo os substratos alimentares considerados como habitats isolados (Hanski, 1987a), e pela presença de duas populações distintas, ou seja, a dos indivíduos adultos, dispersando-se aleatoriamente em busca dos substratos para alimentação e postura, e a dos imaturos, distribuídos nos substratos alimentares por meio da postura de fêmeas adultas (de Jong, 1979; Ives, 1989, 1991).

As posturas de ovos de moscas-varejeiras são geralmente agregadas, em função do comportamento mediado por feromônios, em que fêmeas são estimuladas a depositar seus ovos em local próximo ao de outras fêmeas ovipondo, no mesmo substrato (Norris, 1965; Hanski, 1987a). A agregação dos imaturos nos substratos alimentares refere-se à proporção de indivíduos por unidade de área ou por quantidade de alimento, e pode ser tanto intra como interespecífica (Hanski, 1987a; Rosewell et al., 1990; Ives, 1988, 1991).

A agregação intra-específica tem sido mais estudada em insetos que se utilizam de substratos discretos e efêmeros, o mesmo ocorrendo com a agregação interespecífica, em que se relaciona a agregação das espécies competidoras e sua possibilidade de coexistência (Hanski, 1987a; Kouki \& Hanski, 1995). No entanto, em estudos de competição por recursos alimentares, quando se considera a proporção de indivíduos por grama de alimento, tem-se uma situação em que duas variáveis, ou seja, a densidade de indivíduos e a quantidade de alimento, podem influenciar ao mesmo tempo o resultado da competição.

Dentro desse contexto, o objetivo do presente estudo é observar, em populações experimentais de C. megacephala, se o resultado de competição por alimento é o mesmo em situações em que a proporção de competidores por quantidade de alimento é igual, mas com densidades de competidores e quantidades de alimento proporcionalmente diferentes. Assim sendo, serão analisados os efeitos de diferentes níveis de agregação larval sobre caracteres bionômicos de C. megacephala como peso e fecundidade das fêmeas adultas resultantes, além do índice de investimento reprodutivo, que representa a relação entre o número de ovos produzidos e o peso da fêmea adulta.

\section{MATERIAL E MÉTODOS}

\section{Coleta e manutenção dos espécimens em condições experimentais}

Exemplares de Chrysomya megacephala foram coletados nas proximidades do Departamento de Parasitologia, do Instituto de Biologia da Universidade Estadual de Campinas, em Campinas, São Paulo, Brasil. Foi utilizada como isca, matéria orgânica de origem animal em decomposição, como carcaças de roedores e peixes. Os adultos de ambos os sexos foram coletados com o auxílio de um puçá, identificados e acondicionados em gaiolas com armação de ferro $(30 \times 30 \times 48 \mathrm{~cm})$ e tela de náilon. Foram fornecidos separadamente água e açúcar refinado ad libitum aos insetos, os quais foram considerados como a geração parental do presente estudo.

As gaiolas foram mantidas em sala com temperatura controlada $\left(25 \pm 1^{\circ} \mathrm{C}\right)$, umidade relativa de $60 \pm 10 \%$ e fotoperíodo de 12 horas. Para a formação das gerações seguintes, foram obtidas posturas com a colocação de pequenos frascos de vidro com carne moída putrefata nas gaiolas, como estímulo à oviposição. Para o desenvolvimento gonotrófico das fêmeas das gerações seguintes, foi fornecido figado bovino macerado como fonte protéica, sendo esse fígado fornecido por oito horas nos terceiro e quarto dias após a emergência dos adultos, e por quatro horas nos quinto e sexto dias. 


\section{Formação das densidades larvais}

Para a formação das diferentes densidades larvais de C. megacephala, foram utilizados frascos ( $8 \mathrm{~cm}$ de altura $\mathrm{x} 7 \mathrm{~cm}$ de diâmetro) com quatro diferentes quantidades $(15,30,60$ e $90 \mathrm{~g})$ de dieta artificial (Leal et al., 1982). As densidades foram formadas a partir de larvas da geração $\mathrm{F}_{2}$, e respeitaram para cada quantidade de alimento, cinco diferentes proporções de larvas/g de alimento $(5,10$, 20, 30 e 40 larvas $/ g$ ). Desse modo, foram formadas as seguintes densidades larvais, com dois frascos cada: $75,150,300,450$ e 600 larvas desenvolvendose cada uma em frascos com $15 \mathrm{~g}$ de alimento; 150, $300,600,900$ e 1.200 larvas em frascos com $30 \mathrm{~g} ; 300$, $600,1.200,1.800$ e 2.400 larvas em frascos com $60 \mathrm{~g}$; e, finalmente, $450,900,1.800,2.700$ e 3.600 larvas em frascos com $90 \mathrm{~g}$.

As densidades larvais foram formadas por meio da contagem das larvas imediatamente após sua eclosão a partir do ovo. Os frascos com a dieta artificial foram cobertos com organza e mantidos em câmara climática a $25 \pm 0,2^{\circ} \mathrm{C}, 60 \%$ de UR e fotoperíodo de 12 horas. Imediatamente após a completa exaustão do recurso alimentar pelas larvas, esses frascos foram transferidos para embalagens plásticas maiores, contendo, em sua porção inferior, $5 \mathrm{~cm}$ de maravalha, substrato utilizado para a pupariação. As organzas foram retiradas dos frascos de vidro, para permitir a saída das larvas para o substrato de pupariação. As embalagens plásticas maiores foram, então, cobertas com organza e mantidas nas mesmas condições experimentais.

Após o início da emergência dos adultos, as embalagens plásticas foram transferidas para gaiolas com tela de náilon e tiveram sua organza retirada para a saída dos adultos recém-emergentes. Estes foram alimentados com água e açúcar ad libitum e com fígado bovino fresco macerado. O processo de alimentação com figado foi feito da mesma forma citada anteriormente.

Uma amostra de até 30 fêmeas, com idade de 15 dias após emergência, foi coletada aleatoriamente por frasco, sendo que os indivíduos foram anestesiados em éter sulfúrico, pesados individualmente em balança analítica e dissecados sob estereomicroscópio para a contagem do número de ovos. Para tanto, os ovos foram individualizados com hipossulfito de sódio a $1 \%$. O índice de investimento reprodutivo (Collins, 1980) foi também calculado para cada uma dessas fêmeas, por meio da divisão do número de ovos pelo peso fresco em miligramas da fêmea.

\section{Análise estatística}

A variação nas médias de peso, fecundidade e investimento reprodutivo nas cinco proporções de larvas por grama de alimento foi analisada por meio de dois métodos distintos: teste de comparações múltiplas de Tukey e regressões lineares simples de fecundidade em função do peso da fêmea. Para verificar como a fecundidade varia em função do peso da fêmea, foram calculadas regressões lineares simples para todas as densidades larvais consideradas, $\mathrm{E}(\mathrm{Fec})=\mathrm{b} . \mathrm{P}_{\mathrm{f}}$, em que Fec representa a fecundidade $\mathrm{e} \mathrm{P}_{\mathrm{f}}$, o peso da fêmea. Todas as análises estatísticas foram feitas com o sistema de programas SAS (SAS Institute, 1988).

\section{RESULTADOS}

Os dados de peso fresco das fêmeas, fecundidade e investimento reprodutivo estão representados, respectivamente, nas Tabelas 1,2 e 3 . A análise de variância indicou uma variação significativa nos valores de peso das fêmeas em função da proporção de larvas por grama de alimento $(\mathrm{P}=$ $0,0001 ; \mathrm{F}=149,75)$ e também em função da quantidade de alimento $(\mathrm{P}=0,0001 ; \mathrm{F}=16,38)$. A análise de variância também indicou que a fecundidade $\mathrm{e}$ o investimento reprodutivo diferem significativamente nas diferentes proporções de larvas por grama de alimento $(\mathrm{P}=0,0001$ e $\mathrm{F}=104,46$ para fecundidade; $\mathrm{P}=0,0001$ e $\mathrm{F}=14,19$ para investimento reprodutivo) e nas diferentes quantidades de alimento $(\mathrm{P}=0,002$ e $\mathrm{F}=4,98$ para fecundidade; $\mathrm{P}=0,0001$ e $\mathrm{F}=12,27$ para investimento reprodutivo). Os dados das Tabelas 1 e 2 sugerem uma tendência de queda nos valores de peso e fecundidade das fêmeas, com o aumento da proporção de larvas por grama de alimento, e, conseqüentemente, do nível de competição por alimento.

Na Tabela 4 encontram-se representados os resultados de comparação das médias de peso, fecundidade e investimento reprodutivo, por meio do teste de comparações múltiplas de Tukey. As letras iguais ao lado das médias indicam subconjuntos não significativamente diferentes entre si, ao passo que letras diferentes indicam subconjuntos significativamente diferentes entre si, em nível de significância de 0,05 . 


\section{TABELA 1}

Peso das fêmeas adultas em mg (média $\pm \mathrm{DP} / \sqrt{n}$ ) em cinco proporções de larvas por grama de alimento e quatro quantidades de alimento em C. megacephala, em frascos (1) e (2).

\begin{tabular}{|c|c|c|c|c|c|c|c|c|c|c|}
\hline Proporção & & $\mathbf{5}$ larvas/g & $\boldsymbol{n}$ & $\mathbf{1 0}$ larvas/g & $\boldsymbol{n}$ & $\mathbf{2 0}$ larvas/g & $\boldsymbol{n}$ & $\mathbf{3 0}$ larvas/g & $\boldsymbol{n}$ & $\mathbf{4 0 ~ l a r v a s / g}$ \\
\hline Alimento & & & & & & & & & \\
\hline 15 g (1) & 3 & $92,63 \pm 3,90$ & 6 & $48,73 \pm 4,21$ & 10 & $55,91 \pm 4,89$ & 14 & $50,33 \pm 3,11$ & 30 & $53,56 \pm 1,93$ \\
$(2)$ & 5 & $50,38 \pm 5,84$ & 2 & $54,50 \pm 10,79$ & 6 & $43,92 \pm 5,10$ & 14 & $35,33 \pm 2,02$ & 18 & $38,54 \pm 1,92$ \\
\hline $30 \mathrm{~g}(1)$ & 13 & $66,76 \pm 2,50$ & 30 & $63,71 \pm 2,03$ & 30 & $49,06 \pm 2,12$ & 30 & $61,31 \pm 2,32$ & 30 & $54,65 \pm 2,32$ \\
$(2)$ & 7 & $81,04 \pm 5,48$ & 30 & $70,70 \pm 2,39$ & 30 & $50,13 \pm 1,92$ & 30 & $44,07 \pm 1,19$ & 17 & $23,11 \pm 1,18$ \\
\hline $60 \mathrm{~g}(1)$ & 30 & $79,47 \pm 1,60$ & 30 & $51,20 \pm 1,38$ & 30 & $62,73 \pm 2,37$ & 30 & $43,36 \pm 1,52$ & 30 & $33,50 \pm 1,45$ \\
$(2)$ & 28 & $76,05 \pm 2,88$ & 30 & $53,37 \pm 1,92$ & 30 & $37,69 \pm 1,11$ & 30 & $33,48 \pm 1,36$ & 11 & $28,67 \pm 1,17$ \\
\hline $90 \mathrm{~g}(1)$ & 18 & $76,43 \pm 3,59$ & 30 & $62,91 \pm 2,02$ & 30 & $44,26 \pm 1,68$ & 30 & $64,13 \pm 2,27$ & 30 & $43,06 \pm 1,55$ \\
$(2)$ & 30 & $80,11 \pm 2,52$ & 30 & $57,54 \pm 2,27$ & 30 & $44,51 \pm 1,83$ & 30 & $69,87 \pm 2,02$ & 30 & $39,92 \pm 1,17$ \\
\hline
\end{tabular}

\section{TABELA 2}

Fecundidade representada como o número de ovos (média $\pm \mathrm{DP} / \sqrt{n}$ ) em cinco proporções de larvas por grama de alimento e quatro quantidades de alimento em $C$. megacephala, em frascos (1) e (2).

\begin{tabular}{|c|c|c|c|c|c|c|c|c|c|c|}
\hline Proporção & & $\mathbf{5}$ larvas/g & $\boldsymbol{n}$ & $\mathbf{1 0}$ larvas/g & $\boldsymbol{n}$ & $\mathbf{2 0}$ larvas/g & $\boldsymbol{n}$ & $\mathbf{3 0}$ larvas/g & $\boldsymbol{n}$ & $\mathbf{4 0}$ larvas/g \\
\hline Alimento & & & & & & & & & \\
\hline $\begin{array}{c}15 \mathrm{~g}(1) \\
(2)\end{array}$ & 3 & $340,33 \pm 11,29$ & 6 & $170,83 \pm 30,74$ & 10 & $200,70 \pm 17,99$ & 14 & $182,43 \pm 13,38$ & 30 & $180,13 \pm 5,09$ \\
\hline $30 \mathrm{~g}(1)$ & 13 & $167,20 \pm 22,82$ & 2 & $227,50 \pm 45,50$ & 6 & $187,00 \pm 25,55$ & 14 & $144,86 \pm 5,97$ & 18 & $145,44 \pm 6,40$ \\
$(2)$ & 7 & $258,28 \pm 17,21$ & 30 & $244,43 \pm 8,15$ & 30 & $192,43 \pm 5,52$ & 30 & $155,73 \pm 4,96$ & 17 & $99,29 \pm 5,62$ \\
\hline $60 \mathrm{~g}(1)$ & 30 & $256,53 \pm 5,34$ & 30 & $184,63 \pm 4,23$ & 30 & $221,10 \pm 8,81$ & 30 & $164,70 \pm 6,50$ & 30 & $120,40 \pm 6,01$ \\
$(2)$ & 28 & $243,46 \pm 9,88$ & 30 & $190,93 \pm 6,73$ & 30 & $147,93 \pm 3,44$ & 30 & $125,43 \pm 6,13$ & 11 & $103,00 \pm 3,71$ \\
\hline $90 \mathrm{~g}(1)$ & 18 & $216,72 \pm 15,49$ & 30 & $219,80 \pm 7,50$ & 30 & $154,30 \pm 6,94$ & 30 & $208,40 \pm 7,60$ & 30 & $150,30 \pm 7,60$ \\
$(2)$ & 30 & $294,43 \pm 9,70$ & 30 & $189,17 \pm 5,92$ & 30 & $151,07 \pm 8,40$ & 30 & $191,80 \pm 6,34$ & 30 & $146,27 \pm 3,52$ \\
\hline
\end{tabular}

\section{TABELA 3}

Investimento reprodutivo (média $\pm \mathrm{DP} / \sqrt{n}$ ) em cinco proporções de larvas por grama de alimento e quatro quantidades de alimento em $C$. megacephala, em frascos (1) e (2).

\begin{tabular}{|c|c|c|c|c|c|c|c|c|c|c|}
\hline Proporção & & $\mathbf{5}$ larvas/g & $\boldsymbol{n}$ & $\mathbf{1 0}$ larvas/g & $\boldsymbol{n}$ & $\mathbf{2 0}$ larvas/g & $\boldsymbol{n}$ & $\mathbf{3 0}$ larvas/g & $\boldsymbol{n}$ & $\mathbf{4 0}$ larvas/g \\
\hline Alimento & & & & & & & & & & \\
\hline $15 \mathrm{~g}(1)$ & 3 & $3,68 \pm 0,18$ & 6 & $3,36 \pm 0,35$ & 10 & $3,62 \pm 0,20$ & 14 & $3,65 \pm 0,21$ & 30 & $3,43 \pm 0,09$ \\
$(2)$ & 5 & $3,30 \pm 0,19$ & 2 & $4,17 \pm 0,01$ & 6 & $4,22 \pm 0,37$ & 14 & $4,19 \pm 0,16$ & 18 & $3,83 \pm 0,13$ \\
\hline $30 \mathrm{~g}(1)$ & 13 & $2,50 \pm 0,19$ & 30 & $3,65 \pm 0,11$ & 30 & $3,74 \pm 0,12$ & 30 & $3,47 \pm 0,06$ & 30 & $3,22 \pm 0,08$ \\
$(2)$ & 7 & $3,20 \pm 0,15$ & 30 & $3,52 \pm 0,11$ & 30 & $3,91 \pm 0,09$ & 30 & $3,54 \pm 0,07$ & 17 & $4,31 \pm 0,14$ \\
\hline $60 \mathrm{~g}(1)$ & 30 & $3,24 \pm 0,05$ & 30 & $3,62 \pm 0,05$ & 30 & $3,54 \pm 0,07$ & 30 & $3,82 \pm 0,11$ & 30 & $3,61 \pm 0,11$ \\
$(2)$ & 28 & $3,23 \pm 0,10$ & 30 & $3,60 \pm 0,07$ & 30 & $3,97 \pm 0,08$ & 30 & $3,75 \pm 0,10$ & 11 & $3,63 \pm 0,14$ \\
\hline $90 \mathrm{~g}(1)$ & 18 & $2,81 \pm 0,15$ & 30 & $3,51 \pm 0,08$ & 30 & $3,52 \pm 0,13$ & 30 & $3,25 \pm 0,07$ & 30 & $3,48 \pm 0,11$ \\
$(2)$ & 30 & $3,70 \pm 0,07$ & 30 & $3,35 \pm 0,09$ & 30 & $3,37 \pm 0,11$ & 30 & $2,75 \pm 0,05$ & 30 & $3,70 \pm 0,07$ \\
\hline
\end{tabular}


TABELA 4

Comparação de médias de peso, fecundidade e investimento reprodutivo para cinco proporções de larvas por grama de alimento em $C$. megacephala. As densidades larvais estão representadas entre parênteses. As letras iguais ao lado das médias indicam subconjuntos de médias que não diferem significativamente entre si dentro da mesma proporção.

\begin{tabular}{|c|c|c|c|}
\hline & Peso & Fecundidade & IR \\
\hline & $(450) 78,727 \mathrm{~A}$ & $(450) 265,29 \mathrm{~A}$ & $(75) 3,4461 \mathrm{~A}$ \\
Proporção 5 larvas/g & $(300) 77,816 \mathrm{~A}$ & $(300) 250,22 \mathrm{~A}$ & $(450) 3,3666 \mathrm{~A}$ \\
& $(150) 71,760 \mathrm{AB}$ & $(75) 232,13 \mathrm{AB}$ & $(300) 3,2341 \mathrm{~A}$ \\
& $(75) 66,225 \mathrm{~B}$ & $(150) 198,35 \mathrm{~B}$ & $(150) 2,7443 \mathrm{~B}$ \\
\hline & $(300) 67,202 \mathrm{~A}$ & $(300) 236,87 \mathrm{~A}$ & $(600) 3,6136 \mathrm{~A}$ \\
& $(900) 60,225 \mathrm{~A}$ & $(900) 204,48 \mathrm{~B}$ & $(300) 3,5834 \mathrm{~A}$ \\
Proporção 10 larvas/g & $(600) 52,285 \mathrm{~B}$ & $(600) 187,78 \mathrm{~B}$ & $(150) 3,5646 \mathrm{~A}$ \\
& $(150) 50,213 \mathrm{~B}$ & $(150) 185,00 \mathrm{~B}$ & $(900) 3,4318 \mathrm{~A}$ \\
\hline & $(300) 51,413 \mathrm{~A}$ & $(300) 195,56 \mathrm{~A}$ & $(300) 3,8446 \mathrm{~A}$ \\
& $(1200) 50,210 \mathrm{~A}$ & $(600) 186,77 \mathrm{~A}$ & $(600) 3,8246 \mathrm{~A}$ \\
& $(600) 49,592 \mathrm{~A}$ & $(1200) 184,52 \mathrm{~A}$ & $(1200) 3,7542 \mathrm{AB}$ \\
& $(1800) 44,385 \mathrm{~A}$ & $(1800) 152,68 \mathrm{~B}$ & $(1800) 3,4469 \mathrm{~B}$ \\
\hline & $(2700) 67,000 \mathrm{~A}$ & $(2700) 200,10 \mathrm{~A}$ & $(450) 3,9215 \mathrm{~A}$ \\
& $(900) 52,690 \mathrm{~B}$ & $(900) 182,92 \mathrm{~A}$ & $(1800) 3,7850 \mathrm{~A}$ \\
& $(450) 42,836 \mathrm{C}$ & $(450) 163,64 \mathrm{~B}$ & $(900) 3,5046 \mathrm{~B}$ \\
& $(1800) 38,420 \mathrm{C}$ & $(1800) 145,07 \mathrm{~B}$ & $(2700) 3,0044 \mathrm{C}$ \\
\hline & $(600) 47,927 \mathrm{~A}$ & $(600) 167,12 \mathrm{~A}$ & $(2400) 3,6173 \mathrm{~A}$ \\
& $(1200) 43,243 \mathrm{AB}$ & $(3600) 148,28 \mathrm{~B}$ & $(1200) 3,6145 \mathrm{~A}$ \\
& $(3600) 41,490 \mathrm{~B}$ & $(1200) 147,89 \mathrm{~B}$ & $(3600) 3,5901 \mathrm{~A}$ \\
& $(2400) 32,202 \mathrm{C}$ & $(2400) 115,73 \mathrm{C}$ & $(600) 3,5822 \mathrm{~A}$ \\
\hline
\end{tabular}

Os valores dos coeficientes angulares das regressões $\mathrm{E}(\mathrm{Fec})=\mathrm{b} . \mathrm{P}_{\mathrm{f}}$, em que Fec representa a fecundidade e $\mathrm{P}_{\mathrm{f}}$ o peso da fêmea, seus respectivos erros-padrão e ajustes estão representados na Tabela 5 para as proporções de $5,10,20,30$ e 40 larvas por grama de alimento. As letras iguais ao lado dos coeficientes angulares indicam coeficientes que não diferem significativamente entre si dentro da mesma proporção. As respectivas probabilidades de significância foram as seguintes: 0,0005 (proporção 5), 0,084 (proporção 10), 0,038 (proporção 20), 0,0001 (proporção 30) e, finalmente, 0,0437 (proporção 40).

Os resultados indicam que nas duas proporções extremas de 5 e 40 larvas por grama de alimento, o índice de investimento reprodutivo foi menor para frascos com 30 gramas de alimento, em comparação às outras três quantidades de alimento. Esse mesmo índice foi menor para frascos com 90 gramas (em comparação às outras três quantidades de alimento) nas proporções de 20 e 30 larvas por grama, e não diferiu significativamente em função da quantidade de alimento na proporção de 10 larvas por grama.

\section{DISCUSSÃO}

O principal período de limitação de recursos alimentares em moscas-varejeiras ocorre no estágio larval, e seu modo de competição em substratos discretos e efêmeros é geralmente considerado do tipo por exploração, em que as larvas competem por alimento e cada uma procura ingerir o máximo de alimento no menor intervalo de tempo possível, antes da completa exaustão dos recursos (Bakker, 1961; de Jong, 1976; Levot et al., 1979; Lomnicki, 1988; Goodbrod \& Goff, 1990).

O padrão de distribuição de imaturos nos substratos alimentares, por intermédio da dispersão das fêmeas adultas, pode influenciar o nível de competição por alimento e espaço entre esses imaturos, com conseqüências na viabilidade dos adultos resultantes (de Jong, 1979, 1982; Ives, 1991). 
TABELA 5

Regressão linear de fecundidade em função do peso da fêmea, com os coeficientes angulares, respectivos erros-padrão e ajustes em cinco proporções de larvas por grama de alimento e quatro quantidades de alimento. As letras iguais ao lado dos coeficientes angulares indicam coeficientes que não diferem significativamente entre si dentro da mesma proporção.

\begin{tabular}{|c|c|c|c|c|}
\hline \multicolumn{5}{|c|}{ Proporção 5 larvas/g de alimento } \\
\hline Densidade & Alimento & Coef. angular & Erro-padrão & $r^{2}$ \\
\hline 75 & $15 \mathrm{~g}$ & $3,5479 \mathrm{~B}$ & 0,1339 & 0,99 \\
\hline 150 & $30 \mathrm{~g}$ & $2,7819 \mathrm{~A}$ & 0,1494 & 0,95 \\
\hline 300 & $60 \mathrm{~g}$ & $3,1960 \mathrm{~B}$ & 0,0524 & 0,98 \\
\hline 450 & $90 \mathrm{~g}$ & $3,3716 \mathrm{~B}$ & 0,0873 & 0,97 \\
\hline \multicolumn{5}{|c|}{ Proporção 10 larvas/g de alimento } \\
\hline Densidade & Alimento & Coef. angular & Erro-padrão & $r^{2}$ \\
\hline 150 & $15 \mathrm{~g}$ & $3,7894 \mathrm{~A}$ & 0,2577 & 0,97 \\
\hline 300 & $30 \mathrm{~g}$ & $3,4723 \mathrm{~A}$ & 0,0770 & 0,97 \\
\hline 600 & $60 \mathrm{~g}$ & $3,5686 \mathrm{~A}$ & 0,0447 & 0,99 \\
\hline 900 & $90 \mathrm{~g}$ & $3,3577 \mathrm{~A}$ & 0,0632 & 0,98 \\
\hline \multicolumn{5}{|c|}{ Proporção 20 larvas/g de alimento } \\
\hline Densidade & Alimento & Coef. angular & Erro-padrão & $r^{2}$ \\
\hline 300 & $15 \mathrm{~g}$ & $3,7480 \mathrm{~A}$ & 0,1636 & 0,97 \\
\hline 600 & $30 \mathrm{~g}$ & $3,7069 \mathrm{~A}$ & 0,0674 & 0,98 \\
\hline 1.200 & $60 \mathrm{~g}$ & $3,6072 \mathrm{AB}$ & 0,0579 & 0,98 \\
\hline 1.800 & $90 \mathrm{~g}$ & $3,4292 \mathrm{~B}$ & 0,0819 & 0,97 \\
\hline \multicolumn{5}{|c|}{ Proporção 30 larvas/g de alimento } \\
\hline Densidade & Alimento & Coef. angular & Erro-padrão & $r^{2}$ \\
\hline 450 & $15 \mathrm{~g}$ & $3,7303 \mathrm{~A}$ & 0,1343 & 0,97 \\
\hline 900 & $30 \mathrm{~g}$ & $3,4353 \mathrm{~A}$ & 0,0466 & 0,99 \\
\hline 1.800 & $60 \mathrm{~g}$ & $3,7582 \mathrm{~A}$ & 0,0764 & 0,98 \\
\hline 2.700 & $90 \mathrm{~g}$ & $2,9652 \mathrm{~B}$ & 0,0521 & 0,98 \\
\hline \multicolumn{5}{|c|}{ Proporção $\mathbf{4 0}$ larvas/g de alimento } \\
\hline Densidade & Alimento & Coef. angular & Erro-padrão & $r^{2}$ \\
\hline 600 & $15 \mathrm{~g}$ & 3,4102 B & 0,0695 & 0,98 \\
\hline 1.200 & $30 \mathrm{~g}$ & $3,3007 \mathrm{~A}$ & 0,0737 & 0,98 \\
\hline 2.400 & $60 \mathrm{~g}$ & $3,5657 \mathrm{~B}$ & 0,0946 & 0,98 \\
\hline 3.600 & $90 \mathrm{~g}$ & $3,5563 \mathrm{~B}$ & 0,0699 & 0,98 \\
\hline
\end{tabular}

Vários estudos anteriores com moscas-varejeiras já demonstraram que a redução no tamanho e peso do adulto é uma conseqüência da limitação de alimento e espaço, refletindo a quantidade e qualidade do alimento consumido durante o estágio larval (Kamal, 1958; Sullivan \& Sokal, 1963; Williams \& Richardson, 1983; Slansky \& Rodriguez, 1987; Goodbrod \& Goff, 1990; Reis et al., 1994). Altos níveis de competição larval em populações naturais de moscas-varejeiras geralmente produzem um grande número de indivíduos de pequeno tamanho e baixa fecundidade.

Em comparação com insetos maiores, mas em menor número, aqueles indivíduos têm condições de ocupar grande quantidade de nichos disponíveis, com conseqüências no tamanho e na dinâmica de populações (Sullivan \& Sokal, 1963; Putman, 1977). 
Os resultados do presente estudo demonstraram que houve uma variação significativa nos valores dos caracteres peso, fecundidade e investimento reprodutivo de fêmeas de C. megacephala, com o aumento da proporção de larvas por grama de alimento e, conseqüentemente, do nível de competição por alimento. Além disso, mesmo considerando, para cada quantidade de alimento, as mesmas proporções de larvas por grama, os resultados de peso, fecundidade e investimento reprodutivo são significativamente diferentes nas quatro diferentes quantidades de alimento.

Isso significa que duas ou mais situações com o mesmo nível de agregação larval (proporção de larvas por grama de alimento), mas com densidades larvais e quantidades de alimento proporcionalmente diferentes, não implicam necessariamente no mesmo resultado de competição. Dessa forma, não são necessariamente produzidos, nesse caso, adultos de mesmo peso, fecundidade e investimento reprodutivo.

Uma das possíveis explicações para esse fato seria o comportamento das larvas durante a alimentação, o qual influenciaria a taxa de desenvolvimento das mesmas. Segundo Goodbrod \& Goff (1990), a agregação das larvas de C. megacephala com a conseqüente produção de secreções de enzimas salivares e proteolíticas, aumentaria a eficiência do processo de alimentação e, por conseguinte, aceleraria a taxa de desenvolvimento larval. Por outro lado, aglomerações larvais muito grandes podem prejudicar o processo de alimentação, via aumento da susceptibilidade das larvas aos distúrbios que ocorrem sob severa competição por alimento, tendo como conseqüência a descontinuidade ou até mesmo a completa inibição do processo de alimentação larval (Ullyett, 1950; Bakker, 1961; Levot et al., 1979).

A correlação positiva entre tamanho e fecundidade tem sido demonstrada em vários Diptera, entre eles C. megacephala (Wijesundara, 1957; Von Zuben, 1993; Reis et al., 1994), Lucilia sericata, C. chloropyga e C. albiceps (Ullyett, 1950), Hemipyrellia ligurriens (So \& Dudgeon, 1989a), C. putoria (Reis et al., 1994), Musca domestica (Sullivan \& Sokal, 1963; Ribeiro, 1990), Musca autumnalis (Cilek \& Knapp, 1989), Ephydra cinerea (Collins, 1980) e Drosophila (Bakker, 1961, 1969; Mueller, 1985). Já o investimento reprodutivo apresentou diferentes tendências de variação com o aumento da densidade larval, diminuindo no sarcofagídeo Boettcherisca formosensis (So \& Dudgeon, 1989b) e no califorídeo Calliphora stygia (Williams \& Richardson, 1983), aumentando nos califorídeos Hemipyrellia ligurriens, Calliphora vicina, C. putoria e Lucilia cuprina (Williams \& Richardson, 1983; So \& Dudgeon, 1989a; Reis et al., 1994), e apresentando variação marginalmente significativa em C. megacephala (Reis et al., 1994). Já em Calliphora hilli, o investimento reprodutivo não apresentou variação significativa com o aumento da densidade (Williams \& Richardson, 1983).

Apesar desses estudos terem investigado a variação no peso, fecundidade e índice de investimento reprodutivo das fêmeas em função de crescentes densidades larvais, eles geralmente o fizeram utilizando quantidades fixas de alimento, não investigando a variação desses caracteres dentro de uma mesma proporção de larvas por grama de alimento. Já o presente trabalho, além de analisar a variação desses três caracteres com o aumento das densidades larvais para quatro diferentes quantidades de alimento, também investigou o que ocorre com esses três caracteres dentro de cada nível de agregação, isso em função de diferentes densidades larvais e quantidades de alimento.

Assim sendo, no caso de competição por alimento em moscas-varejeiras, duas situações que correspondam a uma mesma proporção de larvas por grama de alimento, porém com densidades de competidores e quantidades de alimento proporcionalmente diferentes, não permitem necessariamente a mesma possibilidade de acesso ao alimento a todos os competidores. Como esse aspecto terá implicações diretas no resultado final de competição por alimento, a sua não consideração pode prejudicar e/ou comprometer a interpretação desse resultado de competição.

Agradecimentos - O presente trabalho teve o apoio da Fundação de Amparo à Pesquisa do Estado de São Paulo (processos no 94/3851-9, 94/5355-9, 95/8031-2, 95/92999 e 96/9944-4). Os autores agradecem a Sérgio F. dos Reis (Departamento de Parasitologia, IB, Unicamp) e Luiza C. Duarte (Curso de Pós-Graduação em Zoologia, UnespCampus de Rio Claro), pelas sugestões ao manuscrito.

\section{REFERÊNCIAS BIBLIOGRÁFICAS}

BAKKER, K., 1961, An analysis of factors which determine success in competition for food among larvae of Drosophila melanogaster. Arch. Neer. Zool., 14: 200-281. 
BAKKER, K., 1969, Selection for rate of growth and its influence on competitive hability of larvae of Drosophila melanogaster. Neth. J. Zool., 19: 541-595.

CILEK, J. E. \& KNAPP, F. W., 1989, Influence of larval crowding on the adult life history of Musca autumnalis (Diptera: Muscidae). Ann. Entomol. Soc. Am., 82: 346-349.

COLLINS, N. C., 1980, Developmental responses to food limitation as indicators of environmental conditions for Ephydra cinerea Jones (Diptera). Ecology, 61: 650-661.

DE JONG, G., 1976, A model of competition for food. I. Frequency-dependent viabilities. Am. Nat., 110: 1013-1027.

DE JONG, G., 1979, The influence of the distribution of juveniles over patches of food on the dynamics of a population. Neth. J. Zool., 29: 33-51.

DE JONG, G., 1982, The influence of dispersal pattern on the evolution of fecundity. Neth. J. Zool., 32: 130 .

FURLANETTO, S. M. P., CAMPOS, M. L. C., HÁRSI, C. M., BURALli, G. M. \& ISHIHATA, G. K., 1984, Microrganismos enteropatogênicos em moscas africanas pertencentes ao gênero Chrysomya (Diptera: Calliphoridae) no Brasil. Rev. Microbiol., São Paulo, 15: $170-174$.

GOODBROD, J. R. \& GOFF, M. L., 1990, Effects of larval population density on rates of development and interactions between two species of Chrysomya (Diptera: Calliphoridae) in laboratory culture. J. Med. Entomol., 27: 338-343.

GREENBERG, B., 1991, Flies as forensic indicators. $J$. Med. Entomol., 28: 565-577.

GUimarÃES, J. H., PRADO, A. P. \& LINHARES, A. X., 1978, Three newly introduced blowfly species in Southern Brazil (Diptera: Calliphoridae). Rev. Bras. Ent., 22: 53-60.

Guimarães, J. H., PAPAVERO, N. \& PRADO, A. P., 1983, As miíases na região neotropical (identificação, biologia, bibliografia). Rev. Bras. Zool., 1: 239-416.

HALL, M. \& WALL, R., 1995, Myiasis os humans and domestic animals. Adv. Parasitol., 35: 257-334.

HANSKI, I., 1987a, Carrion fly community dynamics: patchiness, seasonality and coexistence. Ecol. Entomol., 12: 257-266.

HANSKI, I., 1987b, Nutritional ecology of dung- and carrion-feeding insects. pp. 837-884. In: F. Slansky \& F. G. Rodriguez (eds.), Nutritional ecology of insects, mites, spiders, and related invertebrates. John Wiley \& Sons, New York.

IVES, A. R., 1988, Aggregation and the coexistence of competitors. Ann. Zool. Fenn., 25: 75-88.

IVES, A. R., 1989, The optimal clutch size of insects when many females oviposit per patch. Am. Nat., 133: 671-687.
IVES, A. R., 1991, Aggregation and coexistence in a carrion fly community. Ecol. Monog., 61: 75-94.

KAMAL, A. S., 1958, Comparative study of thirteen species of sarcosaprophagous Calliphoridae and Sarcophagidae (Diptera) I. Bionomics. Ann. Entomol. Soc. Am., 51: 261-271.

KOUKI, J. \& HANSKI, I., 1995, Population aggregation facilitates coexistence of many competing carrion fly species. Oikos, 72: 223-227.

LAURENCE, B. R., 1981. Geographical expansion of the range of Chrysomya blowflies. Trans. Royal Soc. Trop. Med. Hyg., 75: 130-131.

LEAL, T. T. S., PRADO, A. P. \& ANTUNES, A. J., 1982, Rearing the larvae of the blowfly Chrysomya chloropyga (Wiedemann) (Diptera, Calliphoridae) on oligidic diets. Rev. Bras. Zool., 1: 41-44.

LEVOT, G. W., BROWN, K. R. \& SHIPP, E., 1979, Larval growth of some calliphorid and sarcophagid Diptera. Bull. Ent. Res., 69: 469-475.

LOMNICKI, A., 1988, Population ecology of individuals. Princeton Press, Princeton, 223p.

MUELLER, L. D., 1985, The evolutionary ecology of Drosophila. Evol. Biol., 19: 37-98.

NORRIS, K. R., 1965, The bionomics of blowflies. Ann Rev. Entomol., 10: 47-68.

PUTMAN, R. J., 1977, Dynamics of the blowfly, Calliphora erythrocephala, within carrion. J. Anim. Ecol., 46: 853-866.

REIS, S. F., STANGENHAUS, G., GODOY, W. A. C., VON ZUBEN, C. J. \& RIBEIRO, O. B., 1994, Variação em caracteres bionômicos em função de densidade larval em Chrysomya megacephala e Chrysomya putoria (Diptera, Calliphoridae). Rev. Bras. Ent., 38: 33-46.

RIBEIRO, O. B., 1990, Estudo da competição intraespecífica em Chrysomya putoria (Wiedemann, 1830) (Diptera: Calliphoridae) e Musca domestica (Linnaeus, 1758) (Diptera: Muscidae) em diferentes meios e temperaturas manipulados. Dissertação de Mestrado em Parasitologia. USP, São Paulo, 114p.

ROSEWELL, J., SHORROCKS, B. \& EDWARDS, K., 1990, Competition on a divided and ephemeral resource: testing the assumptions. I. Aggregation. $J$. Anim. Ecol., 59: 977-1001.

SAS INSTITUTE Inc., 1988, SAS/STAT User's Guide. Release 6.03 Edition, Cary, NC.

SLANSKY, F. \& RODRIGUEZ, J. G., 1987, Nutritiona ecology of insects, mites, spiders and related invertebrates: an overview, pp. 1-69. In: F. Slansky \& F. G. Rodriguez (eds.), Nutritional ecology of insects, mites, spiders, and related invertebrates. John Wiley \& Sons, New York.

SO, P.-M. \& DUDGEON, D., 1989a, Variations in the life history parameters of Hemipyrellia ligurriens (Diptera: Calliphoridae) in response to larval competition for food. Ecol. Entomol., 14: 109-116. 
SO, P.-M. \& DUDGEON, D., 1989b, Life-history responses of larviparous Boettcherisca formosensis (Diptera: Sarcophagidae) to larval competition for food, including comparisons with oviparous Hemipyrellia ligurriens (Calliphoridae). Ecol. Entomol., 14: 349356.

SULLIVAN, R. L. \& SOKAL, R. R., 1963, The effect of larval density on several strains of the house fly. Ecology, 44: 120-130.

ULlYETT, G. C., 1950, Competition for food and allied phenomena in sheep-blowfly populations. Phil. Trans. R. Soc. Lond., B234: 77-174.

VON ZUBEN, C. J., 1993, Competição larval em Chrysomya megacephala (Diptera, Calliphoridae): Estimativa de perdas em biomassa e na fecundidade e cálculo de conversão de alimento em biomassa. Rev. Bras. Ent., 37: 793-802.
WELLS, J. D. \& KURAHASHI, H., 1994, Chrysomya megacephala (Fabricius) (Diptera: Calliphoridae) development: rate, variation and the implications for the forensic entomology. Jpn. J. Sanit. Zool., 45: 303309.

WIJESUNDARA, D. P., 1957, The life-history and bionomics of Chrysomya megacephala (Fab.). Ceylon J. Sci., B25: 169-185.

WILLIAMS, H. \& RICHARDSON, A. M. M., 1983, Life history response to larval food shortage in four species of necrophagous flies (Diptera: Calliphoridae). Aust. J. Ecol., 8: 257-263. 